\title{
Renal metastases as the initial presentation of papillary thyroid carcinoma: A case report and literature review
}

\author{
YUNFEI GAO ${ }^{1}$, WEIYE DENG ${ }^{2}$, YANFENG CHEN $^{3}$, YUNPING FAN $^{1}$ and ZHUMING GUO ${ }^{3}$ \\ ${ }^{1}$ Department of Otolaryngology, The Fifth Affiliated Hospital of Sun Yat-sen University, Zhuhai, Guangdong 519020, \\ P.R. China; ${ }^{2}$ Division of Epidemiology, Human Genetics and Environmental Sciences (EHGES), The University \\ of Texas Health Science Center at Houston School of Public Health, Houston, TX 77030, USA; ${ }^{3}$ Department \\ of Head and Neck Surgery, Sun Yat-sen University Cancer Center, State Key Laboratory of Oncology in South China, \\ Collaborative Innovation Center of Cancer Medicine, Guangzhou, Guangdong 510060, P.R. China
}

Received August 10, 2016; Accepted March 7, 2017

DOI: $10.3892 / \mathrm{mco} .2017 .1243$

\begin{abstract}
Distant metastasis of thyroid carcinoma is rare, and it occurs particularly rarely in renal regions, which represent $\sim 3 \%$ of all thyroid distant metastases, with fewer than 30 single case study reports existing in the literature. The present study (and subsequent literature review) of papillary thyroid carcinoma (PTC) reports the case of a 53-year-old male patient who presented with a left renal mass and who had no previous history of thyroid disease. Following renal and thyroid surgery, postoperative pathology confirmed the case to be a follicular variant of PTC, accompanied by renal metastasis. The patient exhibited brain metastasis during a later follow-up. In conclusion, thyroid metastases to the renal site are extremely rare events, particularly for men who present initially with renal neoplasms, which may easily be misdiagnosed as renal cell carcinoma. PTC tends to have a good prognosis; however, when it is accompanied by distant metastasis, the prognosis becomes less favorable.
\end{abstract}

\section{Introduction}

Thyroid carcinoma is the most common endocrine malignancy. The majority of cases are of papillary thyroid carcinoma (PTC), which often has a good prognosis (1). Distant metastases derived from PTC occur in $\sim 4 \%$ of the patients (2). The most common sites are the lungs and bones; however, renal metastasis is rare, with fewer than 30 cases reported in the literature (3), and it is easily misdiagnosed. The present study

Correspondence to: Professor Zhuming Guo, Department of Head and Neck Surgery, Sun Yat-sen University Cancer Center, State Key Laboratory of Oncology in South China, Collaborative Innovation Center of Cancer Medicine, 651 Dongfeng Road, Guangzhou, Guangdong 510060, P.R. China

E-mail: guozhuming58@163.com

Key words: papillary thyroid carcinoma, distant metastasis, renal, follicular variant, prognosis reports a case of PTC with initial presentation of renal metastasis, which was initially misdiagnosed.

\section{Case report}

A 53-year-old man was admitted to the Department of Urology Surgery in Sun Yat-sen University Cancer Center during July 2011 with a complaint of discomfort on the left side of the waist. An occupying lesion in the left kidney was identified by performing computed tomography (CT). The patient had no medical history of renal disease, thyroid disease, neck irradiation, dysphagia or dyspnea. A physical examination at the time of presentation revealed no clear symptoms.

The CT analysis demonstrated a roughly spherical mass $\sim 5 \mathrm{~cm}$ in diameter in the left kidney, and an uneven enhancement in the enhanced scanning images, which was originally considered to be primary renal cell carcinoma, as well as irregular nodular shadows in the left adrenal area and multiple pulmonary nodules. The clinical diagnosis was of primary renal cell carcinoma accompanied by adrenal and bilateral pulmonary metastases; subsequently, the patient was admitted for surgery and underwent a nephrectomy for a renal mass on July 8, 2011.

Histologically, a pathological examination of the left kidney tumor revealed a metastatic follicular variant of PTC. The diagnosis was confirmed by positive immunoperoxidase staining for thyroglobulin, cytokeratin 19 , thyroid transcription factor-1 and vimentin, whereas negative staining of CD10 and thrombopoietin within the tumor cells (Figs. 1 and 2), and the left adrenal schwannoma, were also noted.

A further examination of the thyroid was performed on August 3, 2011. Ultrasonography and CT revealed multiple lesions in both sides of the thyroid accompanied by multiple lesions of the bilateral neck lymph nodes. Total thyroidectomy and bilateral radical neck dissection were performed, and the postoperative pathological results confirmed the follicular variant of PTC in the both sides of the thyroid, with widespread bilateral neck lymph node metastasis, as revealed by the strong positive immunoperoxidase staining of cytokeratin-19, galectin-3 and melanocytes (Fig. 3). The patient underwent radiometabolic treatment with $100 \mathrm{mCi}$ Iodine-131 


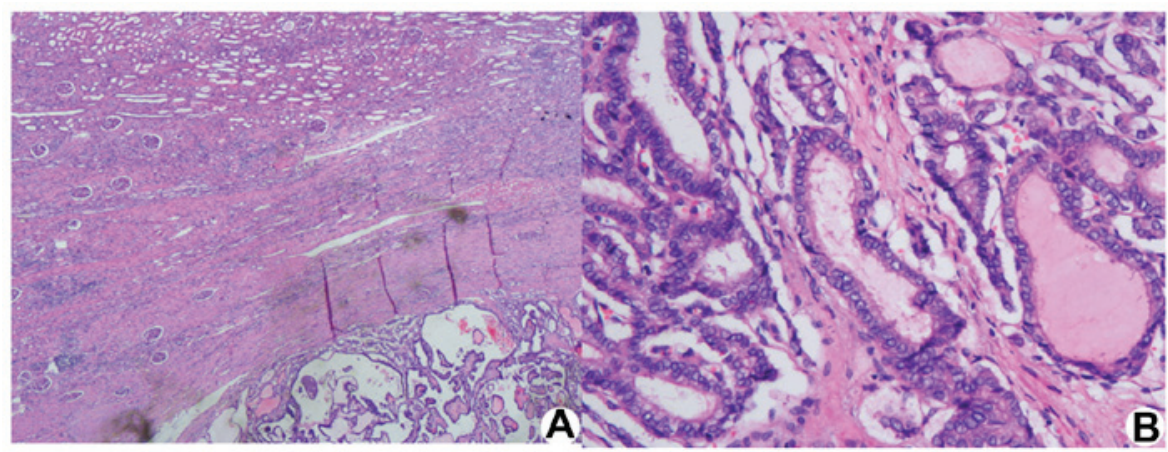

Figure 1. A renal mass with papillary architecture, showing overlapping nuclei with grooves, characteristic of thyroid papillary carcinoma, as revealed by hematoxylin and eosin staining. (A) Magnification, x10; (B) magnification, x20.

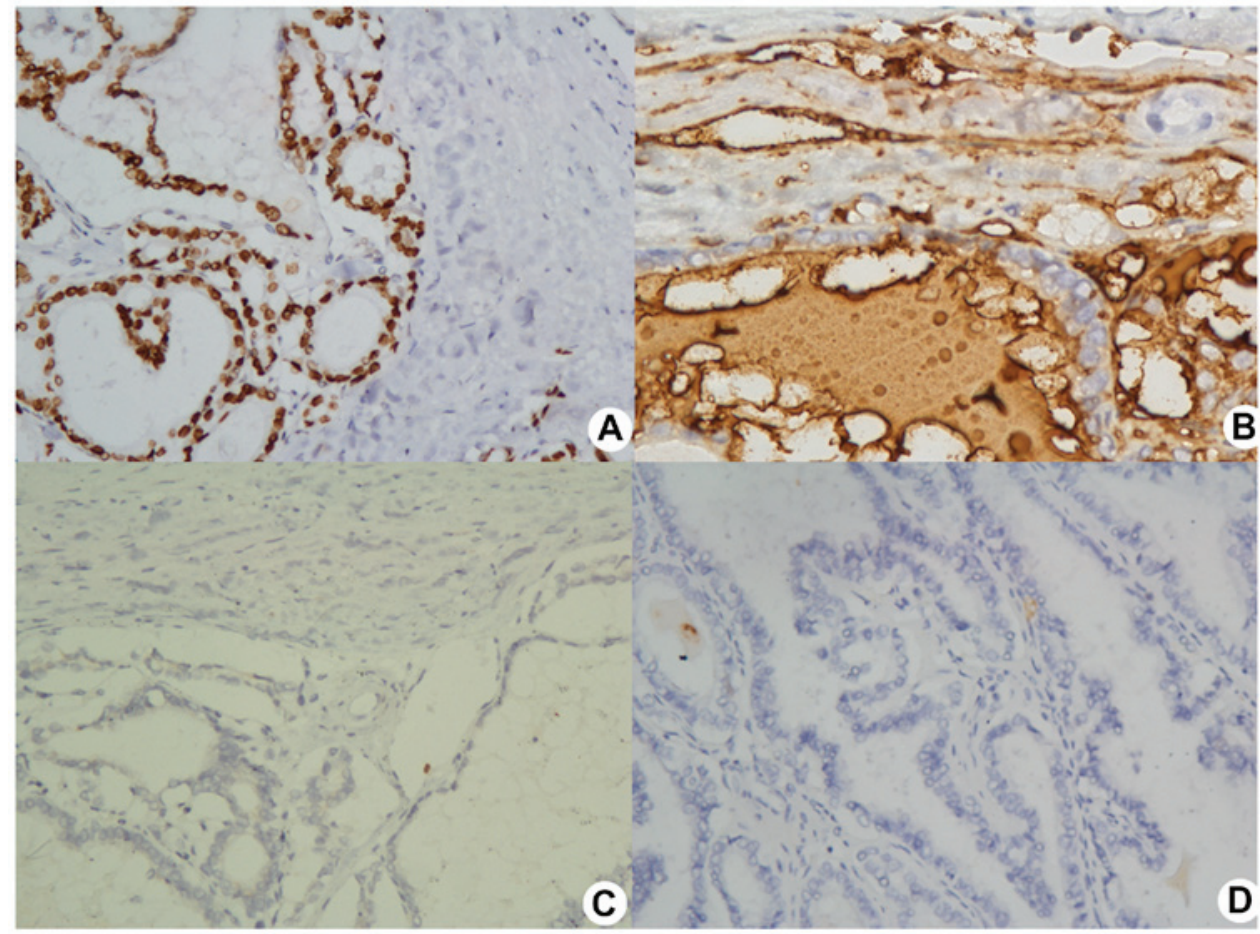

Figure 2. The strong positive immunoperoxidase staining of (A) thyroid transcription factor-1 and (B) thyroglobulin, and negative staining of (C) CD10 and (D) thrombopoietin (as revealed by hematoxylin and eosin staining; magnification, $\mathrm{x} 20$ ).

twice in October 2011 and April 2012, respectively; however, the patient subsequently failed to present at our hospital regularly. On August 21, 2015, the patient attended the hospital to be treated for dyspnea and right-sided hemiparesis. Imaging studies, including CT and magnetic resonance imaging, revealed the presence of multiple nodule metastases in the bilateral pulmonary, bilateral hilar and mediastinal regions, and, of greatest concern, left temporal lobe metastasis (Fig. 4).

\section{Discussion}

Thyroid cancer is one of the fastest growing malignant tumors in the world, and PTC is the most common pathological type of tumor, accounting for $\sim 70 \%$ of cases of thyroid carcinoma, the most common types of which are typical, follicular type and columnar type PTC (4). PTC originates from thyroid follicular epithelial cells, and metastasis occurs most easily to cervical lymph nodes in the neck, which accounts for $\sim 30-90 \%$ of all cases (5). A few cases of PTC that occur are of distant metastasis (ranging from 3.5-3.8\%) (6), and the most common site is the lungs, followed by the bones; however, renal metastasis occurs only rarely, and it is easily misdiagnosed (7). Distant metastases are often associated with a poor prognosis, and the 10-year survival rate is $<50 \%$ (8). Cervical neoplasms (primary tumors or metastasis lymph nodes) are presented frequently as a chief complaint of patients with PTC.

It is rare for patients with PTC to show kidney metastatic lesions at their initial presentation, and the total number of cases reported in the literature is fewer than 30 . The majority of these cases were of female patients $(\sim 80 \%)$, and the cases were concerned with unilateral metastasis. Furthermore, the majority of the cases were of thyroid follicular carcinoma, which accounted for $>50 \%$ of the total, whereas PTC 


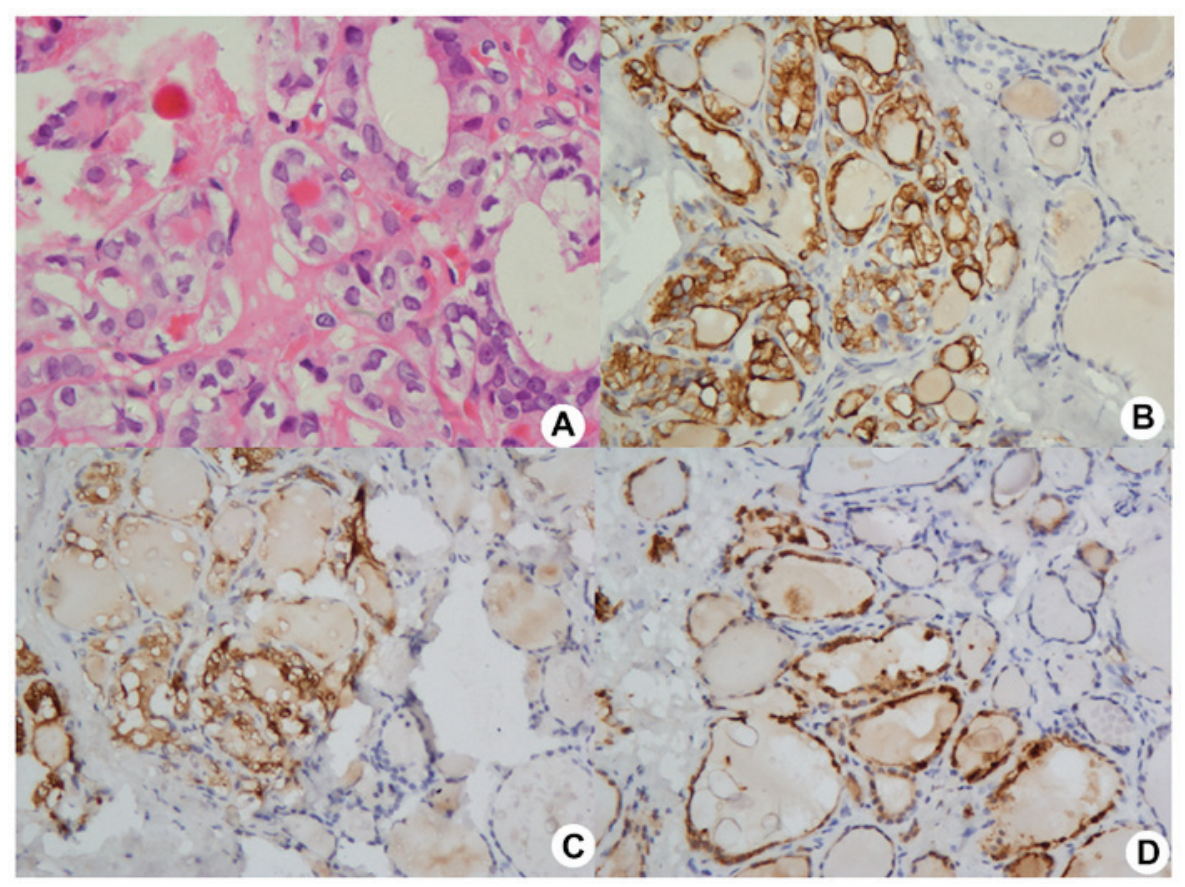

Figure 3. (A) Thyroid nodules, showing the papillary architecture and the intranuclear grooves, as revealed by hematoxylin and eosin staining (magnification, x20). The strong positive immunoperoxidase staining of (B) cytokeratin-19, (C) galectin-3, and (D) melanocytes is also shown.
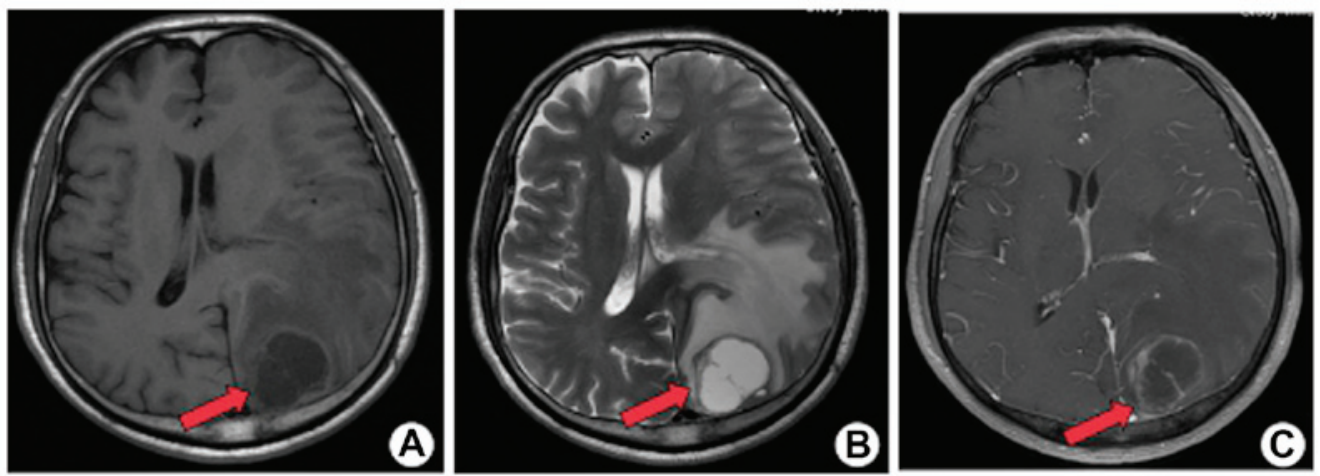

Figure 4. Magnetic resonance imaging, revealing left temporal lobe metastasis. The metastatic tumor shows low signal in (A) T1WI images, and a high signal in (B) T2WI images. (C) Annular enhancement, as revealed by T1WI enhancement scanning, is shown.

accounted for not more than $13 \%(3,9,10)$. Kidney metastases of thyroid carcinoma often occur several years after resection of the thyroid lesions $(11,12)$; initial symptoms with kidney metastasis are rare, and so these cases may easily be misdiagnosed as renal cell carcinoma. The present case was a male patient, whose main complaint was of kidney syndrome, which was misdiagnosed as renal cell cancer, and it was subsequently disclosed to have originated from thyroid carcinoma. The details of the present case differed from those previously reported in the literature (13). Surgery is the first-choice option for PTC, although invasive PTC with distant metastases may result in a limitation of the range of treatment options available, with the notable exception of lung metastases, which are sensitive to Iodine-131 treatment. The pathological diagnosis of the present case was confirmed as renal metastases of the follicular variant of PTC following surgery. Further therapy confirmed the diagnosis as the follicular variant of PTC, accompanied by widespread bilateral cervical lymph node metastasis. A preoperative diagnosis of the follicular variant of PTC is often quite difficult to make, and is often controversial (14).

The prognosis of the follicular variant of PTC is less favorable compared with that of typical PTC, particularly for invasive lesions of the infiltrating type, or with multiple nodules (15). In the present case, the initial diagnosis in July 2011 was of PTC with bilateral neck, bilateral lung and left kidney metastases. Following surgical and radiation therapy, at present, the patient appears to have adrenal gland and brain metastases, which indicate that the condition belongs to the invasive subtype of PTC, and therefore the clinical prognosis for the patient is poor.

In conclusion, renal metastases from PTC with initial renal syndrome occur rarely, and this makes the condition difficult to diagnose and manage. This has been reported in the present case study, and our hope is that this will be helpful for the diagnosis and treatment of renal metastasis from PTC in the future. 


\section{References}

1. Mazzaferri EL and Kloos RT: Clinical review 128: Current approaches to primary therapy for papillary and follicular thyroid cancer. J Clin Endocrinol Metab 86: 1447-1463, 2001.

2. Shaha AR, Shah JP and Loree TR: Differentiated thyroid cancer presen-ting initially with distant metastasis. Am J Surg 174: 474-476, 1997.

3. Varinot J, Ménégaux F, Bitker MO and Compérat E: Renal metastasis from thyroid carcinoma: A case report. Anal Quant Cytopathol Histpathol 36: 46-50, 2014.

4. Lloyd RV, Buehler D and Khanafshar E: Papillary thyroid carcinoma variants. Head Neck Pathol 5: 51-56, 2011.

5. Isaacs JD, McMullen TP, Sidhu SB, Sywak MS, Robinson BG and Delbridge LW: Predictive value of the Delphian and level VI nodes in papillary thyroid cancer. Anz J Surg 80: 834-838, 2010

6. BenbassatCA, Mechlis-Frish S and Hirsch D: Clinicopathological characteristics and long-term outcome in patients with distant metastases from differentiated thyroid cancer. World J Surg 30: 1088-1095, 2006.

7. Mazzaferri EL and Massoll N: Management of papillary and follicular (differentiated) thyroid cancer: New para-digms using recombinant human thyrotropin. Endocr Relat Cancer 9: 227-247, 2002

8. Elisei R, Molinaro E, Agate L, Bottici V, Masserini L, Ceccarelli C, Lippi F, Grasso L, Basolo F, Bevilacqua G, et al: Are the clinical and pathological features of differentiated thyroid carcinoma really changed over the last 35 years? Study on 4187 patients from a single Italian institution to answer this question. J Clin Endocrinol Metab 95: 1516-1527, 2010.
9. Ruggiero FP, Frauenhoffer EE and Stack BC Jr: Papillary thyroid cancer with an initial presentation of abdominal and flank pain. Am J Otolaryngol 26: 142-145, 2005.

10. Song HJ, Xue YL, Xu YH, Qiu ZL and Luo QY: Rare metastases of differentiated thyroid carcinoma: Pictorial review. Endocr Relat Cancer 18: R165-R174, 2011.

11. Malhotra G, Upadhye TS, Sridhar E, Asopa RV, Garde PS, Gawde S and Rangarajan V: Unusual case of adrenal and renal metastases from papillary carcinoma of thyroid. Clin Nucl Med 35: 731-736, 2010.

12. Farina E, Monari F, Tallini G, Repaci A, Mazzarotto R, Giunchi F, Panzacchi R, Cammelli S, Padula GD, Deodato F, et al: Unusual thyroid carcinoma metastases: A case series and literature review. Endocri Pathol 27: 55-64, 2016.

13. Wallander M, Layfield LJ, Jarboe E, Emerson L, Liu T, Thaker H, Holden J and Tripp S: Follicular variant of papillary carcinoma: Reproducibility of histologic diagnosis and utility of HBME-1 immunohistochemistry and BRAF mutational analysis as diagnostic adjuncts. Appl Immunohistochem Mol Morphol 18: 231-235, 2010.

14. Lloyd RV, Erickson LA, Casey MB, Lam KY, Lohse CM, Asa SL, Chan JK, DeLellis RA, Harach HR, Kakudo K, et al: Observer variation in the diagnosis of follicular variant of papillary thyroid carcinoma. Am J Surg Pathol 28: 1336-1340, 2004.

15. DeLellis RA, Lloyd RV, Heitz PU and Eng C (eds): Pathology and genetics of tumours of endocrine organs. In: Kleihues P, Sobrin LH, series editors. World health organization. Classification of Tumours. Lyon, IARC Press, 2004. 